\title{
Mitochondrial dysfunction and redox balance alterations in the development of AD-like pathology in OXYS rats
}

\author{
Mikhail Tyumentsev \\ Molecular mechanisms of aging lab \\ ICG SB RAS \\ Novosibirsk, Russia \\ landselur@bionet.nsc.ru \\ Artyom Gorodetsky \\ Laboratory of magnetic radio \\ spectroscopy \\ NIOCH SB RAS \\ Novosibirsk, Russia \\ gorodaa@nioch.nsc.ru
}

\author{
Natalia Muraleva \\ Molecular mechanisms of aging lab \\ ICG SB RAS \\ Novosibirsk, Russia \\ Myraleva@bionet.nsc.ru \\ Elena Bagryanskaya \\ Laboratory of magnetic radio \\ spectroscopy \\ NIOCH SB RAS \\ Novosibirsk, Russia \\ egbagryanskaya@nioch.nsc.ru
}

\author{
Yulia Polienko \\ Laboratory of Nitrogen Compounds \\ NIOCH SB RAS \\ Novosibirsk, Russia \\ polienko@nioch.nsc.ru
}

\begin{abstract}
This study focuses on the relationship between mitochondrial dysfunction and redox-status in the context of the development of signs of Alzheimer's disease (AD). Mitochondrial dysfunction is considered the missing link between brain aging and $\mathrm{AD}$ [1], the most common type of agerelated dementia worldwide, but the exact causal relationship between mitochondrial dysfunction and the transition from healthy aging to AD remains to be fully understood. Oxidative stress is thought to play a significant role in this mitochondrial dysfunction, leading to cellular damage in redox imbalance. However, the extent of these processes and timing of their occurrence within the scope of $A D$ remain hard to study, especially so in the early, pre-clinical stages of the disease. We explored the mechanisms underlying the disruption of mitochondrial function, their impact on the initiation and progression of pathological molecular cascades of $A D$, and assessed the changes in redox status as one of the main consequences of oxidative stress. This investigation was conducted using senescence-accelerated OXYS rats, which spontaneously develop all major signs of AD and largely reproduce the stages of the disease. We concluded that mitochondrial dysfunction appears to mediate or possibly even initiate AD-like pathology in OXYS rats. Importantly this takes place with no apparent connection to redox imbalance as on both transcriptional and biochemical levels OXYS rats display no significant changes in redox-status and ROS production as compared to controls.
\end{abstract}

Keywords - mitochondria, redox, EPR, Alzheimer's disease, OXYS rats

\section{Introduction}

Growing evidence suggests that mitochondrial dysfunction is an early event in sporadic Alzheimer's disease (AD), but the timeframe within which mitochondrial dysfunction participates in the transition from healthy aging to $\mathrm{AD}$ remains to be studied in depth due to the difficulty of studying early changes in humans and lack of appropriate animal models. Importantly, mitochondria are also the main source of the reactive oxygen species (ROS) and the focal point of many cellular redox reactions. Thus mitochondrial dysfunction is assumed to be directly associated with oxidative stress and redox imbalance which are thought to be tightly connected to aging and thus to age-related diseases.
Using OXYS rats - animal model of sporadic AD exhibiting neuronal and synaptic loss, mitochondrial structural abnormalities, tau hyperphosphorylation, increased $\mathrm{A} \beta$ levels, and $\mathrm{A} \beta$ deposition [2] - we evaluated the interrelationship between mitochondrial dysfunction and the initiation and progression of $\mathrm{AD}$ signs, as well as its connection with redox imbalance in the brain. We assessed the mitochondrial ultrastructure in OXYS rat hippocampal neurons at the age preceding AD-like pathology (from birth to the age of 20 days), age of its manifestation (5 months), and well-pronounced changes (24 months). Ultrastructural alterations were then collated with amounts of proteins of mitochondrial dynamics (mitofusins MFN1 and 2, dynamin1-like protein DRP1) and activity of respiratory chain complexes I, IV, and $\mathrm{V}$ in the mitochondria of prefrontal cortex and hippocampus. To compare the timeline of mitochondrial dysfunction with the timeline of changes in redox balance we have analyzed differential expression of genes related to redox regulation using RNAseq data. Results of this analysis were then verified by measuring the rate of ROS production by brain mitochondria, overall redox status of the brain, NAD/NADH ratio and glutathione content. We have found that signs of mitochondrial dysfunction are present even in the early age preceding the manifestation of AD signs and can exist without pronounced signs of redox dysregulation.

\section{Methods}

Wistar and OXYS rats were obtained from the Breeding Experimental Animal Laboratory of the Institute of Cytology and Genetics, the Siberian Branch of the Russian Academy of Sciences (Novosibirsk, Russia). All animals received food and water ad libitum and were kept at 12/12 light/dark cycle.

In order to assess the extent of mitochondrial dysfunction in different ages, mitochondrial number and morphology was assessed by electron microscopy at the Interinstitutional Shared Center for Microscopic Analysis of Biological Objects (Institute of Cytology and Genetics, Novosibirsk, Russia). Activity of the respiratory chain complexes and the content of the proteins of mitochondrial dynamics were measured by ELISA assays.

Then, to compare the progression of mitochondrial dysfunction against the changes of redox status and redox- 
responding systems, a list of genes related to redox regulating systems was composed using WikiPathways database and relevant terms from GO Ontology database. RNAseq data from the cortex of Wistar and OXYS rats aged 20 days, 12 and 18 months was then used to assess differential expression of genes related to redox status of OXYS rats in different stages of the development of AD signs. Redox status of the brain milieu was measured by EPR spectroscopy using redoxsensitive spin probe methoxycarbonyl-PROXYL. ROS production, glutathione content and NAD/NADH were measured using biochemical assays.

\section{Results}

Electron microscopy shows that on the morphological level, from 5 months on there is marked depletion of neuronal mitochondria. At the same time it should be noted that ultrastructurally damaged mitochondria with rarefied cristae are more abundant in OXYS rats only at the age of 24 months - at the advanced stage of AD signs. Levels of fusion protein MFN1 is higher in OXYS rats which, in conjunction with neuronal mitochondria showing certain degree of enlargement might be a sign of upregulated fusion. However, with age levels of fusion proteins do not increase in OXYS rats - unlike Wistar. OXYS rats display signs of upregulated fission with age. Activity of the respiratory chain complexes is decreased with 20 days old OXYS rats displaying decreased activity of complex IV - which is a known hallmark of brain aging and neurodegeneration [3]. Interestingly, decreased respiratory chain activity in young OXYS rats is not accompanied by $\mathrm{NAD} / \mathrm{NADH}$ ratio shifting towards NADH. In fact, there are signs of a slight shift towards NAD + which might be a sign of compensatory reaction.

Analysis of DEGs related to redox regulation revealed several genes that increase their expression with age both in OXYS and Wistar rats. While there are genes upregulated in OXYS rats compared to Wistar, we have found a number of genes upregulated in Wistar compared in OXYS. Thus, OXYS rats do not present a clear transcriptional profile that would signify a response to oxidative stress or redox imbalance more severe than that in age-matched Wistar rats. Supporting this, we have found no increase in the rate of ROS production in OXYS rats in the ages of manifestation and progression of the disease and only a slight increase while oxidizing a single combination of substrates at the age of 20 days. Importantly, with no increase of ROS production to act upon, the previously shown ability of mitochondrially targeted antioxidant SkQ1 to alleviate signs of the AD [4,5] might be due to changes in signal pathways in response to the treatment. The data pointing at lack of redox imbalance in OXYS rats are further corroborated by the fact that redox balance of the brain milieu has displayed an expected shift to the pro-oxidative state from 20 days to 12 months of age in both Wistar and OXYS rats with no interstrain differences. Additionally, we have observed an increase of glutathione content occurring from the age of 5 months to the age of 18 months, possibly as a part of compensatory response, which was slightly less pronounced in OXYS rats.

\section{Conclusion}

Here we present the timeline of the decline of mitochondrial function in OXYS rats. We show that mitochondrial dysfunction precedes other signs of $\mathrm{AD}$ and further progresses along with the progression of the disease itself. Interestingly, redox imbalance in the brain of OXYS rats appears to be largely uncoupled from the progression mitochondrial dysfunction and the development of AD signs, and thus might be seen as optional rather than inherent part of these conditions.

\section{ACKNOWLEDGMENT}

This study was supported by the Russian Foundation for Basic Research (Grant No. 17-03-01132-a) and Russian Science Foundation (Grant No. 19-13-00235).

\section{REFERENCES}

[1] Grimm, Amandine, Kristina Friedland, and Anne Eckert. "Mitochondrial dysfunction: the missing link between aging and sporadic Alzheimer's disease." Biogerontology, vol. 17, no. 2, pp. 281-296, 2016.

[2] Stefanova, Natalia A., Natalia A. Muraleva, Elena E. Korbolina, Elena Kiseleva, Kseniya Yi Maksimova, and Nataliya G. Kolosova. "Amyloid accumulation is a late event in sporadic Alzheimer's diseaselike pathology in nontransgenic rats." Oncotarget, vol. 6, no. 3, pp. 1396-1413, 2015

[3] Tyumentsev, Mikhail A., Natalia A. Stefanova, Natalia A. Muraleva, Yulia V. Rumyantseva, Elena Kiseleva, Valentin A. Vavilin, and Nataliya G. Kolosova. "Mitochondrial dysfunction as a predictor and driver of Alzheimer's disease-like pathology in OXYS rats." Journal of Alzheimer's Disease, vol 63, no. 3, pp. 1075-1088, 2018

[4] Stefanova, Natalia A., Anzhela Zh Fursova, and Nataliya G. Kolosova. "Behavioral effects induced by mitochondria-targeted antioxidant SkQ1 in Wistar and senescence-accelerated OXYS rats." Journal of Alzheimer's Disease, vol 21, no. 2, pp. 479-491, 2010

[5] G Kolosova, Nataliya, Mikhail A Tyumentsev, Natalia A Muraleva, Elena Kiseleva, Anton O Vitovtov, and Natalia A Stefanova. "Antioxidant SkQ1 alleviates signs of Alzheimer's disease-like pathology in old OXYS rats by reversing mitochondrial deterioration." Current Alzheimer Research, vol 14, no. 12, pp. 12831292,2017 